ks. Radosław Kimsza

Politechnika Biatostocka

DOI: $10.15290 /$ std.2017.03.06

\title{
LA DONNA - SOMIGLIANTE ALLO SPIRITO SANTO E RIVOLTA ALLA THEOTOKOS
}

\section{KOBIETA - PODOBNA DO DUCHA ŚWIĘTEGO I ZWRÓCONA KU THEOTOKOS W WIZJI P. N. EVDOKIMOVA}

Artykuł prezentuje myśl jednego z najwybitniejszych współczesnych teologów prawosławia w obszarze antropologii teologicznej P. N. Evdokimova. Ukazuje on fenomen kobiety widzianej przez Teologa jako podobnej do Ducha Świętego, bo uświęcającej, ożywiającej i rodzącej. Swój archetyp kobieta odnajduje w Theotokos - Matce Boga i Pneumatoforze - Nosicielce Ducha Świętego ucząc się ontycznej czystości, która przygotowuje człowieka do przyjęcia Boga i stania się Jego mieszkaniem.

Słowa kluczowe: Evdokimov, prawosławie, antropologia teologiczna, kobieta, Theotokos, obraz i podobieństwo Boga.

\section{Introduzione}

Negli ultimi anni sembra risvegliarsi in occidente l'interesse per l'oriente cristiano e, allo stesso tempo, gli orientali sono attenti ai valori maturati durante i secoli nella formazione culturale dell'ovest; gli occidentali rivolgono lo sguardo all'est ed esaminano il suo patrimonio spirituale. Le cause di questo processo sono diverse. Le ritroviamo anche nella storia. E così, cominciando dall'inizio di questo secolo, la difficile situazione politica dei paesi orientali slavi - in particolare della Russia che, in seguito alla rivoluzione dell'ottobre 1917 è diventata il centro propulsore del comunismo e delle sue idee - ha costretto molti creatori della cultura, nel senso ampio della parola, a lasciare 
la patria e cercare all'estero i luoghi della realizzazione e dell'espressione dei propri valori. Questo fatto, pur avendo avuto le sue dolorose conseguenze, ha permesso di conservare la ricchezza dei valori spirituali, purtroppo quasi totalmente distrutti nella loro terra originaria, e condividerli con l'uomo occidentale, introducendo così l'oriente nell'occidente. Il destino degli artisti, degli scrittori, dei pittori, è stato condiviso anche dai teologi ortodossi. Perseguitati per la fede da loro professata, all'estero cercavano di creare centri di pensiero teologico ortodosso ${ }^{1}$. Tra di loro, accanto ai più grandi teologi russi - ortodossi di questo secolo², troviamo Pavel Nikolaevič Evdokimov (1901-1970). Dagli inizi, la scuola in cui si formava il suo pensiero teologico rappresentava due diverse correnti teologiche. La Prima seguiva il metodo originario della teologia orientale, quello dei Padri; l'altra, cercando risposte alle domande che pone la teologia, si rivolgeva ai concetti filosofici, in particolare a quelli sofiologici di V. Solovev ${ }^{3}$. Che strada sceglie Evdokimov? Affascinato dalla persona di Sergio Bulgakov e dal suo pensiero, scrive che nella sua scuola "bisognava coltivare l'«istinto dell'ortodossia», immergersi nel pensiero dei padri, vivere la liturgia, «consumare il fuoco» eucaristico, capire l'icona, scoprire l'escatologia, la meta - storia"'. Questi elementi, da lui stesso indicati, sono diventati il fondamento della sua idea teologica. Nelle sue ricerche Evdokimov attinge, quindi, al tesoro della spiritualità dell'Oriente Cristiano, esamina il fondo del patrimonio accumulato nella tradizione della Chiesa. Non lo fa però come un semplice ricercatore, un teologo - scientifico. Il suo pensiero è vissuto e immerso nella quotidianità della vita. E benché non lo sappiamo dall'autobiografia, che mai è stata scritta, lo dicono le testimonianze degli altri. Per i suoi figli era "un vero padre spirituale" ${ }^{5}$ "un «rivelatore» che invitava a discendere nella profondità segreta dell'essere" ${ }^{6}$. Per gli altri, egli con il suo pensiero, era "adoratore in spirito, che ha lottato contro la lettera che uccide. Adoratore in verità, che

1 Si tratta dell'Istituto San Sergio di Parigi, fondato nel 1924, che è tuttora il centro scientifico della teologia ortodossa nell'ovest e dell'Istituto San Vlodymyr di New York.

2 Si trattadiN. Berdiaev, S. Bulgakov, G. Florovskij, V. Lossky, J. Meyendorff, A. Schmemann.

3 Por. A. Kniazeff, L'Iinstitut Saint-Serge. De l'Academie d'autrefois au rayonnoment d'aujourd'hui (Le Point Théologique 14), Paris 1974, p. 118.

$4 \quad$ P. Evdokimov, Le Buisson ardent (Bible et vie chretienne), Paris 1981, p. 15.

$5 \quad$ N. Pecheff-Evdokimov, Mon père, Contacts 23 (1971), p. 227.

$6 \quad$ M. Evdokimov, Un réfugié russe, Contacts 23 (1971), p. 233. 
cercava in tutte le cose ciò che è autentico"7, "l'uomo giusto al posto giusto, l'uomo giusto nel tempo giusto"8 e confessore della verità nella carità'.

La lettura dei saggi di Evdokimov permette di osservare che l'Oriente non è unica fonte della sua teologia. Egli cerca l'ispirazione anche nella tradizione occidentale e la trova nei Padri dei primi secoli del Cristianesimo, nei mistici e santi fino al Concilio Vaticano II e nell'insegnamento di Papa Paolo VI. Così cerca di trovare i punti di incontro dell'unica Chiesa di Cristo, rivelando la sua particolare grandezza quale "maestro ecumenico, espressione della teologia e della Chiesa prima della divisione" ${ }^{10}$, "testimone meraviglioso della vera Chiesa"11.

Il pensiero di Evdokimov è permeato dall'insegnamento dei Padri della Chiesa. Essi, nello sviluppo degli argomenti teologici, partivano sempre dall'uomo, in quanto Dio, di per sé stesso invisibile, lo si può conoscere solo attraverso la sua immagine. L'uomo rappresenta l'immagine più perfetta dipinta da Dio stesso, a Sua immagine e somiglianza. In quest'affermazione si trova la risposta al perché la teologia creata dai primi scrittori cristiani è antropologica ${ }^{12}$. I pensatori religiosi russi, a cui appartiene Evdokimov, preferivano approfondire il tema da un altro punto di vista: la conoscenza dell'uomo è condizionata al riferimento della sua esistenza a Dio. Questo modo di procedere implica che l'antropologia è teologica ${ }^{13}$. E', quindi, l'ambiente in cui è cresciuta la coscienza teologica del nostro Autore che ha creato l'espressione per cui "l'uomo stesso si pone come teologia vivente, come luogo teologico per eccellenza" ${ }^{14}$.

Lo sguardo generale sulla storia della teologia permette di distinguere due profili prevalenti di concezione dell'uomo. Il primo statico, che descrive la persona umana nella sua costituzione essenziale nel momento della creazione. Laltro dinamico, che racconta la storia della salvezza e spiega il fenomeno umano quale risultato di una sequela d'avvenimenti. Tale antropologia, particolarmente sensibile ai temi dell'origine, della colpa e della redenzione, aperta all'orientamento cristocentrico ed escatologico, rappresenta l'uomo

7 L. Gillet, Homelie. Paul Evdokimov adorateur en esprit et en vérité, Contacts 23 (1971), p. 8.

8 W. A. Visser't Hooft, Messages, Contacts 23 (1971), p. 216.

9 C. Westphal, Messages, Contacts 23 (1971), p. 218.

$10 \quad$ N. A. Nissiotis, Messages, Contacts 23 (1971), p. 219.

11 C. Charlier, Une amitié en esprit, Contacts 23 (1971), p. 240-247.

12 T. Špidlik, Prefazione, [in:] M. Tenace, Dire l'uomo, t. II, Dall'immagine di Dio alla somiglianza. La salvezza come divinizzazione, Roma 1997, p. 9.

13 Cf. ibidem.

14 Por. P. Evdokimov, L'Orthodoxie, Bibliothèque Théologique, Delachaux et Niestlé, Neuchatel: Paris 1959, p. 57. 
come immagine di Dio, deturpato nella somiglianza e rinnovato dall'unica vera immagine, Cristo, "Prototipo"15.

\section{La diversità della rivelazione di Dio nell'uomo}

La creazione dell'uomo nella dualità maschile e femminile contribuisce, ad approfondire le relazioni intratrinitarie e si pone come punto di partenza per ogni rappresentazione e comprensione ulteriore dell'analogia maschile - femminile ${ }^{16}$. Un'analisi dei primi due capitoli della Genesi pone subito in evidenza l'importanza attribuita dall'autore sacro alla creazione dell'uomo e della donna. Il valore della reciprocità integrativa dell'uomo e della donna espresso nell'"assai buono" (Gen 1,31) del primo racconto, e riaffermato nel "non è bene che l'uomo sia solo" (Gen 2, 18) del secondo racconto si presenta come la magna carta della teologia dell'uomo e della donna, teologia che evidenzia la diversità della rivelazione di Dio nella persona umana. In quest'aspetto biblico, a partire dalla teologia dell'immagine e somiglianza, Evdokimov, congiungendo la natura divina con quell'umana, si domanda come le relazioni intratrinitarie possano riflettersi nella condizione umana. Emerge così il fondamento trinitario della struttura umana, sia in quanto persona che in quanto espressa nella dualità maschile e femminile. La reciprocità delle nature umano - divina si riflette dirittamente nella natura umana che è investita, secondo Evdokimov, nel suo essere maschile e femminile, di un valore carismatico. Quest'indicazione c'indirizza ai carismi che sono inscritti nella natura dell'uomo e della donna. In essi si rivela non solo la distinzione tra i due esseri creati, ma anche i compiti da fare nella realizzazione delle vocazioni che sorgono dalla sessualità umana ${ }^{17}$.

Le osservazioni di Evdokimov permettono affermare che il carisma della donna esiste prima di tutto nell'essere. La sua presenza nel mondo è unificatrice. Con l'insieme della sua natura ella conserva, protegge, integra; la delicatezza del suo essere, in se stessa s'indirizza verso la santità. Nel suo essere è inscritta la vocazione alla realizzazione dell'annunzio di Dio, pronunciato in dialogo con

15 Por. G. Giannini, Il problema antropologico: linee e sviluppo storicospeculativo dai presocratici a s. Tommaso, Roma 1965, p. 58; W. Telfer, The Birth of Christian Anthropology, The Journal Theological Studies 13(1962), p. 347.

16 Evdokimov analizza la creazione dell'uomo, nella distinzione tra maschile e femminile, innanzitutto nelle opere relative al sacramento del matrimonio: Le Mariage, Sacrement de l'Amour, Lyon 1947 e Le Sacrement de l'Amour. Le mystère conjugal à la lumière de la Tradition Orthodoxe, Paris 1962. I punti del riferimento sull'argomento li troviamo anche nel libro La Femme et la Salut du Monde. Etude d'anthropologie chrétienne sur les charismes de la femme, Tounai - Paris, 1958.

17 Por. Le Mariage, Sacrement de l'Amour, 47. 
il serpente dopo la caduta dell'uomo: "Io porrò inimicizia tra te e la donna, tra la tua stirpe e la sua stirpe: questa ti schiaccerà la testa e tu le insidierai il calcagno" (Gen 3, 15). Evdokimov dirrà: "La donna trafigge il dragone, e non con qualche atto, ma col suo semplice essere, con la sua purità. Proprio questa santità dell'essere è ai demoni mortalmente insopportabile. (...) La donna, estatica si volge verso l'interno del suo essere, o semplicemente dell'essere. L'elemento femminile agisce al livello della struttura ontologica, non è quindi il verbo ma l'«esse» (essere), il seno della creatura" ${ }^{\prime 18}$.

\section{Chiamata all'essere somigliante allo Spirito Santo}

Evdokimov osserva che la costituzione della donna, i carismi inscritti nella sua natura la mettono come "somigliante allo Spirito Santo". La realtà contenuta in quest'affermazione rivela la sua vera e giusta vocazione nel mondo. Ella "non è chiamata ad una semplice cooperazione di sforzi o ad una collaborazione pragmaticamente utile e giustificata, ma alla creazione insieme con l'uomo della realtà assolutamente nuova del maschile e del femminile che vanno a formare il corpo del Sacerdozio Regale" ${ }^{19}$. Non si tratta qui la presenza collaboratrice nel senso di servire. La figura della donna è distinta dall'uomo ma complementare ad esso. Essa esprime la persona, il suo dinamismo di trasparenza e di comunione trinitariamente fondata: "è chi sta faccia a faccia; di fronte al figlio di Dio si pone la figlia di Dio; l'uno completa l'altra; s. Paolo dice che «né l'uomo può fare senza la donna, né la donna senza l'uomo nel Signore» (1 Cor 11, 11)"20. La creazione della donna "da Adamo" e come "aiuto" dell'uomo, non importa "una creatura meno perfetta". La creazione del femminile significa piuttosto che l'uomo senza la donna non può vivere la propria verità di creatura. Insieme al carattere maschile, quello femminile, somigliante allo Spirito Santo, aiuta l'uomo a ricordare che la sua presenza è un soffio d'amore.

La somiglianza allo Spirito Santo dona alla donna una particolare sensibilità che rappresenta una specifica caratteristica della natura femminile. Quest'attributo si trasferisce nella sua vita in cui ogni elemento religioso trova

$18 \quad$ P. Evdokimov, Le Sacrement de l'Amour, p. 46.

19 Ibidem, p. 19.

20 Ibidem, p. 20. Per approfondire il tema: M. Rupnik, Dire l'uomo l'uomo, t. 1: Persona, cultura della Pasqua, Roma 1996, p. 93-94. L'Autore conferma che la creazione di Eva da Adamo rappresenta una visibile oggettivazione di Adamo. Eva è creata come „aiuto” perché nell'atto della creazione „diviene l'altro di Adamo”. Rappresenta l'aiuto di Adamo perché quasi non cada "con il tentatore nella perversione egoista" e non si chiude "in un guscio isolato, incamminandosi così verso la morte". 
il suo riflesso. Perciò l'iconografia mostra la sua esistenza nelle immagini della preghiera, dell'adorazione e della lode al Creatore. Questa visione permette, ad Evdokimov, di avvicinare l'immagine della donna a quella dell'Eucaristia. L"'epiclesi" dello Spirito Santo durante la liturgia dell'Eucaristia unisce, trasforma, porta la comunione nel corpo. La vocazione della donna all'essere somigliante al Paraclito implica che ella possiede la capacità di riunire, di fare uno. "Per la sua struttura religiosa, la donna è l'atto di integrazione vivificante" osserva Evdokimov ${ }^{21}$. Non solo questo avvicina la donna all'aspetto eucaristico. L'Autore mira al fondo del mistero nascosto nella femminilità e cerca di collegare due realtà radicate nell'agire delle due donne - Eva e Maria Vergine - la causa e il compimento delle promesse divine: "Gli elementi più decisivi del destino umano si attualizzano nell'elemento religioso per eccellenza; l'umanità comunica eucaristicamente con il male nella donna e la promessa della salvezza affonda le sue radici sempre nella donna - spiga di grano: la donna «ti schiaccerà il capo» (Gen 3, 15), la donna partorirà il Salvatore"22.

Tra i carismi della donna, nella sua struttura fisica adattata a partorire e spirituale, è inscritta la maternità. Listinto materno, inserito nella capacità fisiologica della donna, secondo le osservazioni del nostro Autore, è condizionato dai suoi valori spirituali: "Dal suo spirito materno che le viene la facoltà fisiologica e la corrispondenza anatomica" ${ }^{23}$. Il carisma della maternità fa parte della natura d'ogni donna, senza distinzione tra la vergine e la sposa. Questa condizione è così radicata nella personalità femminile che diventa un istinto che necessita di una realizzazione fisica e spirituale: "Il suo carisma della «maternità» interiorizzata ed universale spinge ogni donna verso l'affamato e il bisognoso e sottolinea mirabilmente l'essenza femminile: vergine o sposa, la donna è madre «in eternum». Le componenti del suo animo la predispongono a covare tutto ciò che cresce lungo il suo cammino, a scoprire anche nell'essere più virile e forte un bambino debole e indifeso ${ }^{24}$.

La Vergine Maria, Nuova Eva, nella sua immacolata concezione tramite lo Spirito Santo, conosce nel profondo il senso della maternità. Il carisma della maternità affidato alla donna, con l'annunciazione dell'Angelo e in conseguenza la nascita di Gesù, cambia la sua natura. Ella diventa Madre conservata nella verginità: "Nuova Eva in Cristo, Maria reca la verità circa la natura umana; la Chiesa la dichiara eternamente «vergine», vergine in essenza e perciò madre. E' perché di regola la verginità fiorisce in maternità

\footnotetext{
21 P. Evdokimov, La Femme et la Salut du Monde, p. 157.

22 Ibidem, p. 158

23 Ibidem, p. 12.

24 Ibidem, p. 41.
} 
che ogni donna è chiamata a ciò che é materno", così Evdokimov commenta la maternità che non si oppone alla verginità 25 .

Evdokimov osserva che l'istinto materno è più forte di quello paterno, e lo inserisce nella concezione di matriarcato. E' interessante che l'istinto paterno della prima Persona della Trinità nella Sacra Scrittura è espresso con le immagini della maternità (cfr. Ga 4, 19). Questa forza, che viene dal dono così caratteristico per la donna, costruisce il fondamento religioso di ogni essere umano. Il nostro Autore nota che "il principio religioso, nell'umano, si esprime attraverso la donna (...) nella sua purezza, é nell'«anima» e non nell' «animus»" ${ }^{26}$. In ciò risulta il collegamento della maternità con la paternità. La sua espressione è evidente in modo particolare, nota Evdokimov, nella relazione tra Theotokion e Theotokos. I testi liturgici suggeriscono che nella Vergine si rivela la figura umana della Paternità divina ${ }^{27}$. "Alla paternità divina come carattere distintivo dell'essenza del Padre che genera il Figlio e fa procedere da sé lo Spirito Santo, risponde dirittamente la maternità femminile come specificità religiosa della natura umana" ${ }^{28}$, riflette Evdokimov.

\section{Rivolta alla Theotokos}

La donna trova il suo archetipo nella Madre di Dio - Theotokos - Pneumatofora - la portatrice dello Spirito Santo ${ }^{29}$. Il suo libero e confidente Fiat all'iniziativa di Dio ha rivelato la sua castità degna di collaborare nell'opera della redenzione dell'uomo e la volontà aperta ai progetti divini. Questo "si" della Vergine, raggiunge il suo culmine nel mistero della croce. "Dalla ferita della Vergine, dallo Spirito e dal sangue è nato l'Archetipo femminile. Per la Vergine, il suo ministero di donna comincia nell'istante dell'Annunciazione, ma archetipicamente risale e si radica nella croce" afferma Evdokimov ${ }^{30}$.

La presenza della Theotokos è santificante, rivela la pienezza nel senso archetipico, diventa continua rivelazione della santità e del rendimento di

$25 \quad$ Ibidem, p. 43.

26 Ibidem, p. 152.

27 Ci si riferisce al frammento del terzo degli otto toni della liturgia dei vespri domenicali, relativamente alla parte chiamata dogmatikon: "Tu hai generato il Figlio senza padre, questo Figlio che il Padre ha generato senza madre, prima che i secoli fossero". Cf. ibidem.

28 Ibidem, p. 156.

29 Il nostro Autore dedica a questo tema uno dei capitoli del suo libro fondamentale riguardante la teologia della donna La donna e la salvezza del mondo sotto il titolo La Theotokos: Archetipo del femminile. Cf. ibidem, p. 211-224. La partecipazione della Vergine all'opera di redenzione è alquanto sottolineata nei testi liturgici della festa dell'Annunciazione. 
gloria a Dio. La Vergine Maria, che ha generato la Luce e che rappresenta il primo essere umano divinizzato, illumina il mondo e con questa luce indica la strada verso la salvezza. In essa si realizza la divinizzazione, destinata da Dio a tutto l'universo. Grazie alla santità e divinità della Vergine, che è un riflesso della divina ipostasi di Gesù Cristo, il mondo diventa una nuova creatura. La divinizzazione della Vergine esiste quindi nella sua maternità divina: "LAgiofania e la Dossofania, manifestazione della santità e della gloria, la Vergine testimonia il progetto di Dio sulla creatura: la perfetta unione dell'umano e del divino nel primo essere umano deificato. Il mondo è stato creato per deificare la creatura, e nella Vergine questo destino universale è pre - realizzato, perché Maria è la sua prefigurazione archetipica" ${ }^{31}$.

L'umanità partecipa alla natura divina attraverso la carne di Maria offerta al Cristo. Se quindi Gesù è "la Via, la Verità e la Vita" $(G v 14,6)$ la sua Madre è la "Odigitria" 32 , la guida per eccellenza sulle vie della salvezza. "La Vergine è la «prima»: precede l'umanità e tutti la seguono. Genera il Cammino e pone se stessa come «buona direzione», una «colonna di fuoco» che porta verso

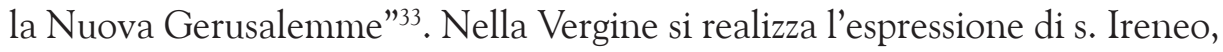
ammirata dai Padri, è spesso citata da Evdokimov: "Dio si è fatto uomo perché l'uomo diventasse dio".

Quando parliamo di Theotokos come l'archetipo dobbiamo riferirci secondo Evdokimov, al Sacerdozio Regale Femminile. In questo contesto l'Autore pone l'accento sulla natura della donna e afferma che il servizio che caratterizza il sacerdozio dell'ordinazione è inscritto nel suo essere umano e non riguarda le funzioni: "Il ministero d'ordine non è compreso fra i suoi carismi e rappresenterebbe quindi un tradimento del suo essere" 34 .

Il sacerdote ministro capeggia il popolo cristiano verso la salvezza, alla liturgia celeste. Là non esistono più funzioni. Il sacerdote è unico - il Cristo. Lui "celebrerà la liturgia celeste, ma a capo del popolo, del sacerdozio regale, resta la Theotokos che lo esprime come «esse» del Regno stesso: la santità «in aeternum» e l'epitalamio del «Sanctus»" ${ }^{35}$.

$31 \quad$ Ibidem, 213.

32 La parola Odigitria deriva dal greco hodégeo e significa guidare. In Bisanzio è stata creata un tipo d'icona che rappresenta la Madonna come la Guida sulla strada della vita. La Vergine del tipo Hodigitria, "colui che mostra la via", rappresenta il dogma cristologico e mostra suo Figlio, Colui che è la via. Sul braccio sinistro porta il bambino che benedice e con la destra indica il Salvatore. Por. P. Evdokimov, L'Art de l'Icône. Théologie de la Beauté, Paris 1970, p. 220.

34 Ibidem, p. 214-215.

35 Ibidem, p. 216. 
Ogni donna contemplando il mistero della presenza della Theotokos scopre in essa l'archetipo della castità ontologica, la vocazione alla purificazione del mondo, l'essere il roveto ardente. Il carisma della castità, come quello della maternità è inserito nella natura d'ogni donna, sia sposa, sia vergine. "Si sottolinea così il carisma specificamente femminile della purezza che ha il compito di eliminare l'inclinazione all'iniquità che intacca e corrompe l'ontologia umana" indica Evdokimov ${ }^{36}$ determinandola con il greco termine sophrosyn. Esso esprime non solo la castità ontologica ma nello stesso tempo l'integrità conforme alla Sapienza. Così la castità non è solo un attributo che caratterizza la natura femminile, ma rivela tramite la Vergine e la sua purezza materna la più potente filantropia divina ${ }^{37}$.

Rivolta alla Theotokos, la donna ritrova lo spirito del servizio, che sorge dall'essere la Madre di colui che "non é venuto per essere servito, ma a servire" (Lc 1,38). L'attributo che accompagna la sua verginità e maternità deve essere inteso nel senso di un ministero d'intercessione per tutta la Chiesa. La Vergine Maria per la sua divina maternità è dimora dello Spirito Santo: "Lo Spirito Santo scenderà su di te" (Lc 1, 35). La presenza del Paraclito riguarda quindi anche il femminile, collega ogni donna con gli avvenimenti santificanti e trasformanti del Consolatore. Evdokimov osserva che "il femminile, quando è imperniato sul proprio essere, è posto sotto il segno della Natività e della Pentecoste, della generazione della nuova creatura e del nuovo eone"38.

Infine rivolgersi alla Theotokos significa imparare a dire a Dio il fiat che nasconde la forza unificatrice, il fiat che una volta pronunciato fa entrare sulla strada della santità, alla salvezza nell'unità. Questo fiat contiene quindi l'aspetto ecumenico ed è espresso nell'umile preghiera, nell'adorazione della presenza divina che può diventare l'inizio della riconciliazione. E non solo questo. La donna chiamata a stare accanto all'uomo, con tutti i suoi carismi e la sua femminile sensibilità religiosa, deve diventare un segno della vita radicata nei valori, imparati rivolgendo continuamente lo sguardo all'Archetipo, alla Vergine - Theotokos. "Alla donna spetta il compito di correggere lo zelo maschile che molto spesso e sempre di più tende alla profanazione dei misteri e alla perdita dei valori spirituali" ${ }^{39}$.

La Vergine è stata chiamata a riportare la vittoria contro il male, tramite la sua purezza. La sua continua apertura all'agire dello Spirito Santo ha permesso di accogliere il Redentore. Evdokimov sottolinea come ogni donna possieda

\footnotetext{
$36 \quad$ Ibidem, p. 217.

37 Ibidem.

38 Ibidem, p. 221-222.

39 Ibidem, p. 223.
} 
la capacità di generare Dio nelle anime umane: "La donna ha innanzitutto questo carisma materno di generare il Cristo nelle anime degli uomini. (...) La salvezza verrà solo dalla santità e questa, nelle condizioni della vita attuale, è più intima alla donna (...), ogni donna ha un'intimità innata, quasi una complicità, con la tradizione, la continuità della vita; la donna, nella santità, con la forza dell'umiltà è particolarmente capace di avvicinare l'essenza e l'esistenza perché «l'umiltà è l'arte di trovarsi esattamente al proprio posto»"40.

\section{Conclusione}

Il pensiero teologico di Evdokimov riguardante la donna rivela il "feminismo" che non distrugge l'essere umano e in stesso tempo la civilizazzione umana ma fa vedere la belezza della donna che supera la semplice estetica e diventa la belezza divina che non passa mai. La teologia della donna creata da Evdokimov attinge dalla sua esperienza personale - le doppie nozze, la vita coniugale e la paternità nell'ordine della natura. Così per il feminismo contemporaneo e in un certo senso la perdita della verità sull'essere feminile la teologia di Evdokimov può diventare l'indicazione alla riscoperta dei veri e giusti valori della donna e rinnovare il suo posto nel mondo e in stesso tempo il compimento della sua vocazione cristiana - diventare sempre di più somigliante allo Spirito Santo con il punto di riferimento alla Theotokos.

Parole chiave: Evdokimov, ortodossia, antropologia teologica, donna, Theotokos, immagine e somiglianza di Dio.

\section{Bibliografia:}

1. Evdokimov P., L'Art de l'Icône. Théologie de la Beauté, Paris 1970.

2. Evdokimov P., Le Buisson ardent (Bible et vie chretienne), Paris 1981.

3. Evdokimov P., La Femme et la Salut du Monde. Etude d'anthropologie chrétienne sur les charismes de la femme, Tounai - Paris, 1958.

4. Evdokimov P., Le Mariage, Sacrement de l'Amour, Lyon 1947.

5. Evdokimov P., L'Orthodoxie, Bibliothèque Théologique, Delachaux et Niestlé, Neuchatel: Paris 1959.

6. Evdokimov P., Le Sacrement de l'Amour. Le mystère conjugal à la lumière de la Tradition Orthodoxe, Paris 1962.

7. Charlier C., Une amitié en esprit, Contacts 23 (1971), p. 240-247.

8. Giannini G., Il problema antropologico: linee e sviluppo storicospeculativo dai presocratici a s. Tommaso, Roma 1965.

9. Gillet L., Homelie. Paul Evdokimov adorateur en esprit et en vérité, Contacts 23 (1971), p. 5-9.

$40 \quad$ La Femme et la Salut du Monde, 224. 
10. Kniazeff A., L'Iinstitut Saint-Serge. De l'Academie d'autrefois au rayonnoment d'aujourd'hui (Le Point Théologique 14), Paris 1974.

11. Nissiotis N. A., Messages, Contacts 23 (1971), p. 39-51.

12. Pecheff-Evdokimov N., Mon père, Contacts 23 (1971), p. 225-229.

13. Rupnik M., Dire l'uomo l'uomo, t. 1: Persona, cultura della Pasqua, Roma 1996.

14. Špidlik T., Prefazione, [in:] M. Tenace, Dire l'uomo, t. II, Dall'immagine di Dio alla somiglianza. La salvezza come divinizzazione, Roma 1997, p. 9-10.

15. Telfer W., The Birth of Christian Anthropology, The Journal Theological Studies 13(1962), p. 347-354.

16. Visser't Hooft W. A., Messages, Contacts 23 (1971), p. 216-217.

17. Westphal C., Messages, Contacts 23 (1971), p. 217-218. 